\title{
Wavelet Transform and Convolutional Neural Network Based Techniques in Combating Sudden Cardiac Death
}

\author{
Wanzita Shilla, Xiaopeng Wang \\ Lanzhou Jiaotong University \\ School of Electronics and Information Engineering \\ E-mail: wangxiaopeng@mail.lzjtu.cn \\ Correspondence Author: 2018b28012@stu.lzjtu.edu.cn
}

Received October 2, 2021; Revised November 3, 2021; Accepted December 5, 2021

\begin{abstract}
Sudden cardiac death (SCD) is a global threat that demands our attention and research. Statistics show that $50 \%$ of cardiac deaths are sudden cardiac death. Therefore, early cardiac arrhythmia detection may lead to timely and proper treatment, saving lives. We proposed a less complex, fast, and more efficient algorithm that quickly and accurately detects heart abnormalities. Firstly, we carefully examined 23 ECG signals of the patients who died from SCD to detect their arrhythmias. Then, we trained a deep learning model to auto-detect and distinguish the most lethal arrhythmias in SCD: Ventricular Tachycardia (VT) and Ventricular Fibrillation (VF), from Normal Sinus Rhythm (NSR). Our work combined two techniques: Wavelet Transform (WT) and pre-trained Convolutional Neural Network (CNN). WT was used to convert an ECG signal into scalogram and CNN for features extraction and arrhythmias classification. When examined in the MIT-BIH Normal Sinus Rhythm, MIT-BIH Malignant Ventricular Ectopy, and Creighton University Ventricular Tachyarrhythmia databases, the proposed methodology obtained an accuracy of $98.7 \%$ and an F-score of 0.9867 , despite being less expensive and simple to execute.
\end{abstract}

Keywords: Sudden Cardiac Death, Arrhythmia, Electrocardiogram, Wavelet Transform, Convolutional Neural Network.

\section{INTRODUCTION}

Sudden cardiac death (SCD) kills between 300,000 and 400,000 persons in the United States each year [1]. Sudden cardiac death has surged dramatically in China, the world's most populous country. SCD claims the lives of around 544,000 Chinese people each year, making China the country with the highest number of sudden cardiac deaths in the world [2],[3],[4]. Africa continent is believed to be facing the same problem whereby sudden cardiac death shortens many lives [5],[6]. Unfortunately, there is no precise online data regarding the demises [7].

A heart rhythm abnormality, commonly known as cardiac arrhythmia, causes sudden cardiac death [8]. Cardiac arrhythmia is when the heart loses 
its capacity to pump blood to the brain, lungs, and other body regions. As a result, a patient dies within a short period after experiencing symptoms related to a loss of heart function. SCD occurs unexpectedly after the manifestation of severe symptoms. The time classification can be divided into three groups. (i)Instant death or immediate death: The patient dies within seconds and minutes after the onset of illness. (ii)Very sudden death: Death occurs in one hour after the manifestation of symptoms. (iii)Sudden death: Death occurs within 1 to 24 hours after symptoms appear. Most of the victims do not manifest any early warning symptoms before the sudden cardiac death; only a few of them experience some pains in their chest and have the family member dies due to sudden death. Therefore, making it hard to predict. However, we can still combat this problem by identifying people at risk the most and giving them close attention for early and proper treatment. According to previous research, people with heart arrhythmias had a higher risk of sudden mortality than the general population [9],[10]. Not all cardiac arrhythmias, however, are fatal [11]. Thus, why our work focused only on distinguishing the most lethal cardiac arrhythmias in SCD, which are Ventricular fibrillation (VF) and Ventricular tachycardia (VT) [12]. Studies show that about $85 \%$ of sudden cardiac deaths are due to VT and VF, and the remaining percentage is bradyarrhythmia [13].

Ventricular tachycardia is recognized as a life-threatening arrhythmia with a 150 to 250 beats per minute ventricular rate. When the speed is above $250 \mathrm{bpm}$ is referred to as ventricular flutter and termed as slow ventricular tachycardia when the rate is less than $150 \mathrm{bpm}$. It usually originates in the ventricles, therefore, has no PRI. This rhythm is not easy to distinguish between the QRS waveform and the T wave; the QRS complexes are vast and inexplicable with the duration of 0.12 seconds, $\mathrm{P}$ waves do not precede the QRS wave. Ventricular fibrillation is also lethal arrhythmia whereby the heart beats very fast and out of rhythm. The heart rate in the VF waveform cannot be calculated because P waves, QRS complexes, and PRI waves are absent. Instead, there are chaotic electrical impulses to the ventricles and incomplete ventricular contractions, resulting in fibrillation of the heart instead of contraction. Normal sinus rhythm is a regular beat with a heart rate of 60 to 100 beats per minute, and the conduction of all beats is usually done throughout the ventricles. All of the complexes are of the same size and shape. The P-QRS-T complex is regular and complete. It is also characterized by constant PR, RR, and PP waves; the P wave is smooth, uprighted, rounded, and in each QRS waveform, there is one $P$ wave, while the $T$ waves are uprighted in the lead II. The values of PR and QT are about 0.1 to 0.2 seconds and 0.36 to 0.44 seconds, respectively. The PRI is constant and has a matter of 12 to 20 seconds.

The QRS complex of a regular beat is generally from 0.4 to 0.12 seconds. Figure 1 is the ECG waveform representation of normal sinus rhythm, ventricular tachycardia, and ventricular fibrillation electrocardiogram waveforms. Despite both VF and VT being dangerous in the human body, they 
can be treated if detected earlier. Therefore, early detection of cardiac arrhythmias is vital.

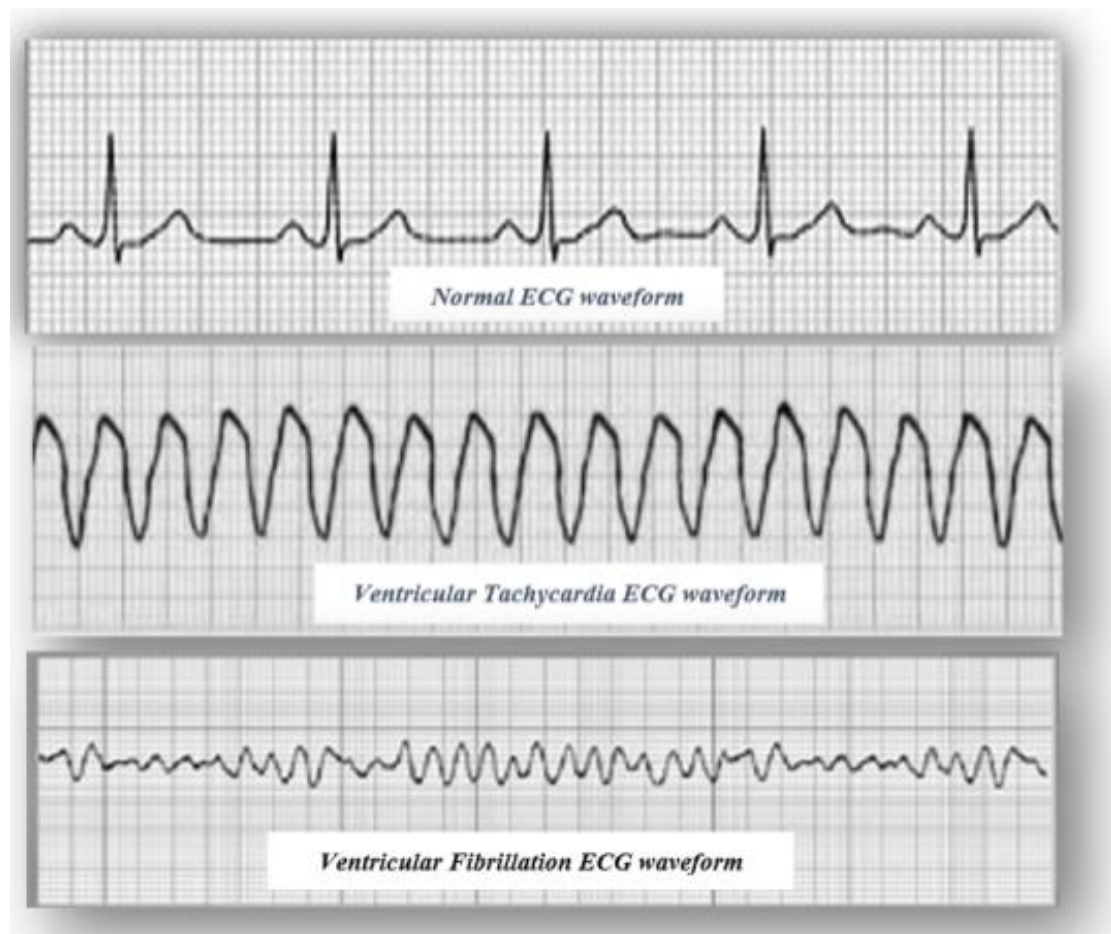

Figure 1. Type of heart rhythms for classification using the proposed method.

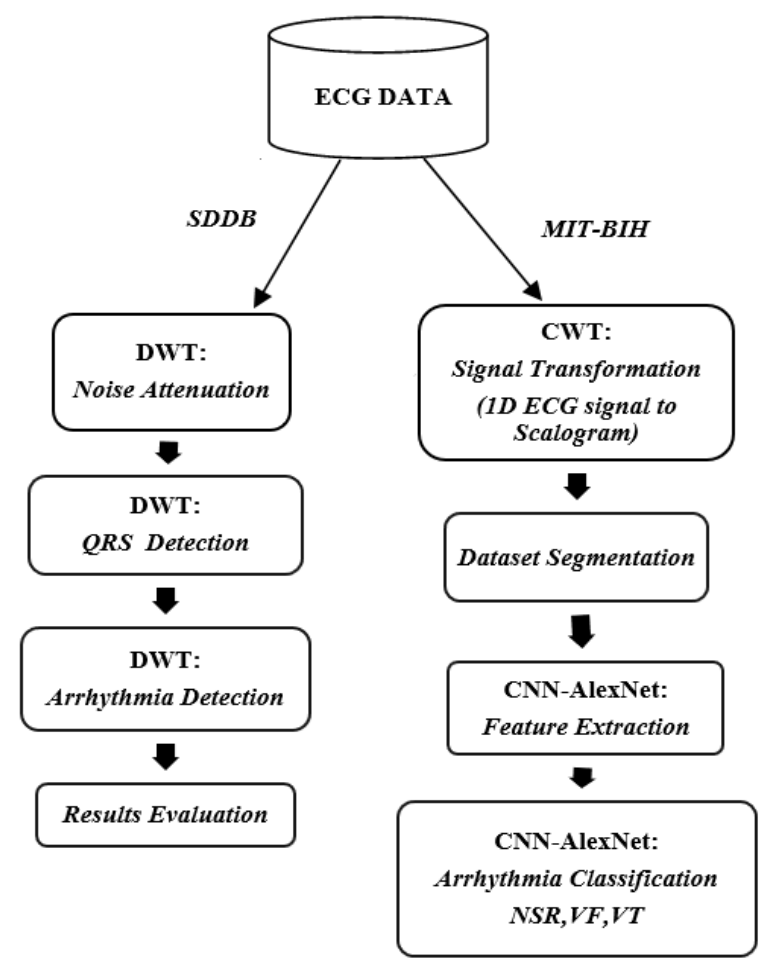

Figure 2. The block diagram of the proposed method. 
Our work combined Wavelet Transform and a pre-trained Convolutional Neural Network. Figure 2 briefly explains the general process of this paper. This work is divided into two main sections as mentioned below: (i) Applying Discrete Wavelet Transform (DWT) technique to distinguish normal heart rhythm from abnormal ones based on QRS complex theory. (ii) Combining Continuous Wavelet Transform (CWT) and a deep Convolution Neural Network (CNN) to detect and distinguish two cardiac arrhythmias (VT and VF) from Normal Sinus Rhythm (NSR).

\section{RELATED WORKS}

The prior studies proposed different techniques that automatically detect heart diseases. [14] combined three methods: CNN, LSTM, and CLSTM, to classify cardiac arrhythmias. The Convolutional Recurrent Neural Network method was implemented by Xiong et al. [15] to detect heart arrhythmias.

In recent years, the deep Convolutional Neural Network technique has mainly been used in medical image processing, and it has made an enormous contribution [16],[17],[18]. CNNs do not require any other feature extraction techniques like Association Rules, Correlation-based feature subset selection, Rough Set theory, Principal Component Analysis. Instead, the CNNs themselves can study various filter extraction features and perform autoclassification of the feature vectors. But building the CNN model from scratch is not easy, and its training process requires a massive amount of data. Thus, a pre-trained deep learning method was introduced, which is simple in design, costless, yet quickly detecting heart abnormalities accurately [19]. For example, Mashrur et al. [20] applied a transfer learning neural networks method for automatic recognition of Atrial Fibrillation arrhythmia, and the performance accuracy of $97.9 \%$ and F-score of $98.82 \%$ was achieved.

Alaskar et al. [21] employed a pre-trained AlexNet Convolutional Neural Network to differentiate standard PCG signals from abnormal ones. The 2016 PhysioNet/CinC Challenge Database assessed the accuracy of the whole system, which was $87 \%$ when AlexNet was used to extract features while Support Vector Machine was applied to classify signals. The 85\% accuracy was obtained when AlexNet was used in the end-to-end learning method.

\section{ORIGINALITY}

Firstly, we investigated the ECG signals of 23 patients who died from sudden cardiac death. The Discrete Wavelet Transform method and some MATLAB codes were applied to create an algorithm that distinguished each patient's ECG signal as normal or abnormal. The experimental results uncovered that out of 23 patients who died from SCD, 19 patients had Ventricular fibrillation (VF), 4 patients had Ventricular tachycardia (VT), while none of them manifested the normal heart rhythm (NSR); this motivated us to design a system that will detect and distinguish those two cardiac arrhythmias (VF, VT) from healthy heartbeat (NSR). Therefore, as proposed above, we trained a deep Convolutional neural network that detected and classified the most lethal arrhythmia in sudden cardiac death. But building a deep neural 
network from scratch requires an extensive database and expertise for appropriate convergence. The only way to overcome this drawback is by using transfer learning, also known as the fine-tuning method. Thus, we applied a transfer learning method using the AlexNet-Convolutional Neural Network algorithm instead of building a model from scratch. The databases used to test our deep model are MIT-BIH Normal Sinus Rhythm, MIT-BIH Malignant Ventricular Ectopy, and Creighton University Ventricular Tachyarrhythmia Database, which are available on the physionet website. All the experiments performed in this paper were executed in MATLAB 2018b software. To our understanding, no study has employed the proposed approaches and the same databases used in this research to categorize VT, VF, and NSR till now. As a result, direct comparison is hard.

\section{SYSTEM DESIGN}

Regarding methodology, this work is divided into two main sections.

\subsection{Discrete Wavelet Transform Method}

This section examined the patients who died due to sudden cardiac death. The main goal was to distinguish the normal rhythms from abnormal ones in those victims. The 23 ECG records were selected for this study. We all know that the ECG signals are always affected by artefacts since they are tiny in amplitude and their sensitivity behaviour. Thus, any small amount of noise mixed with an ECG signal can alter it and lead to incorrect clinical interpretation and improper treatment. Therefore, the filtering process remains essential as the ECG data corrupted with noise must be filtered or discarded [22]. The most common types of noise in the ECG signal are electrode motion artefacts, powerline interference, baseline wanders, and electromyographic (EMG) noise [23]. This work applied the discrete wavelet transform decomposition method to remove the unwanted noise from the required original signals. In addition, the wavelet bandpass filtering was implemented to eliminate the wavelet coefficients having a high frequency and low frequency of ECG signals which are not needed.

\subsubsection{QRS detection using Discrete Wavelet Transform}

Three waveforms make up the QRS complexes: Q, R, and S. We only concentrated on detecting the R peaks of each ECG signal and using them to determine heart rate in this study. A real rate in beats per minute or the RR interval in milliseconds can be used to describe heart rate. We need to know the RR distance between the two QRS complexes to determine the patient's heart rate. The RR interval can be estimated by detecting the $\mathrm{R}$ peak of the first QRS complex cycle and the value of the R peak of the next cycle, as illustrated in figure 3 . The heart rate in beats per minute is then calculated by dividing 60 by the value of the RR interval [24]. MATLAB2018b software deconstructed the selected ECG signals to the fourth level using discrete wavelet transform and symlet4. All R Peaks-related frequencies were kept, while all other frequencies were deleted. As indicated in Figure 4, the estimated Coefficient 
a 4 was ignored, as were the details $\mathrm{d} 1$ and $\mathrm{d} 2$. Only the coefficients $\mathrm{d} 3$ and $\mathrm{d} 4$ were taken into account for the bandpass filter effect. The desired signal was obtained by taking the inverse wavelet transform action and considering the details coefficients $\mathrm{d} 3$ and $\mathrm{d} 4$. The heart rate of each ECG signal was then calculated using the obtained value of the $\mathrm{R}$ peaks with a defined time interval [25]. Two types of cardiac arrhythmias occur when the heart rate (HR) does not fall within the usual range of 60 to 100 beats per minute: bradyarrhythmia (HR 60bpm) and tachycardia (HR >100bpm). However, we cannot determine if a cardiac rhythm is normal or irregular based on heart rate. Other conditions, on the other hand, must be followed as well. In this work, we considered all four criteria physicians used to distinguish between normal and abnormal ECG signals: heart rate, pathway, speed, and origin [26]. The experimental results of this section are explained in detail in the next section of the experiment and analysis.

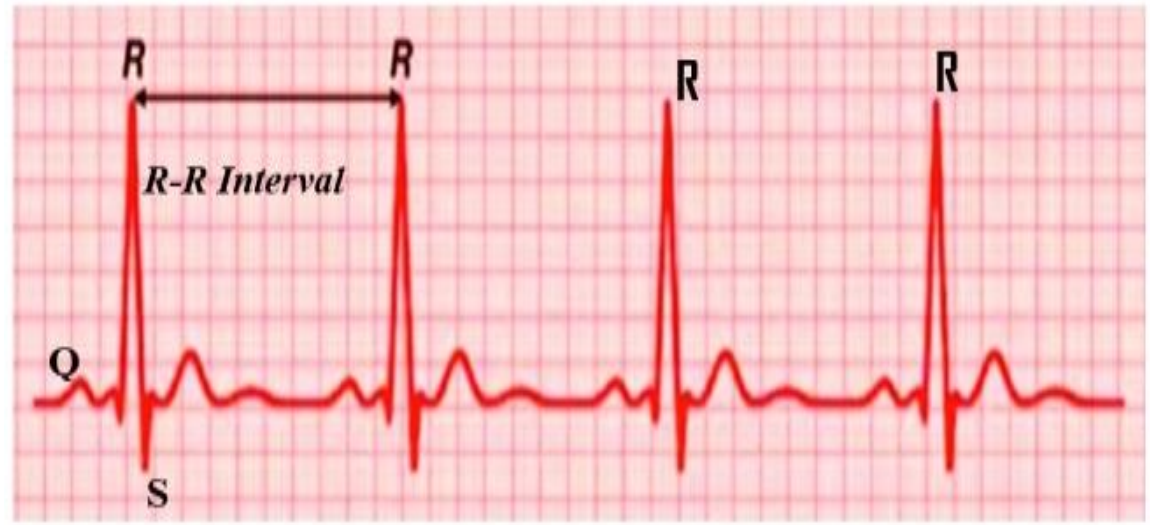

Figure 3: An ECG waveform showing the QRS complex and RR interval.
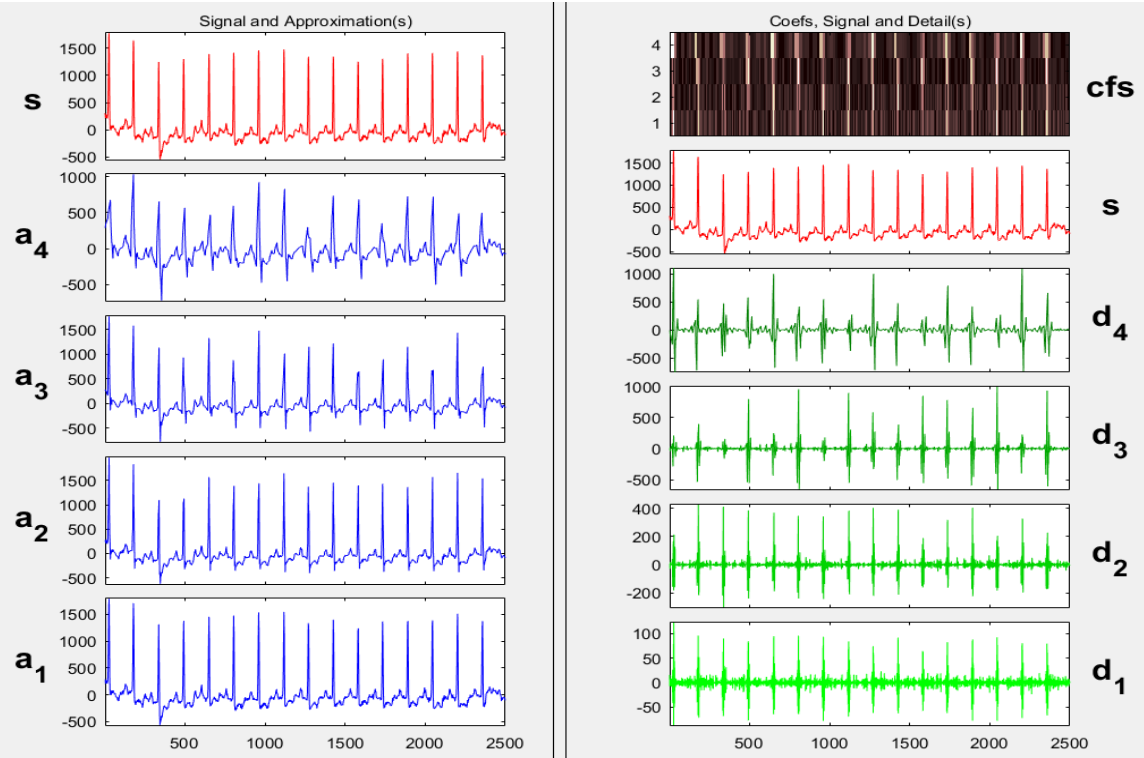

Figure 4. Four levels of decomposition of ECG signal as executed in MATLAB software using Discrete Wavelet Transform. 


\subsection{Wavelet Transform and Convolutional Neural Network Method}

This section combined Wavelet Transform (WT) and Deep Convolutional Neural Network (CNN). WT was used to convert an ECG signal into scalogram and CNN for features extraction and arrhythmias classification.

\subsubsection{Data Transformation using Continuous Wavelet Transform}

Since the ECG signal is a one-dimensional time series (1-D signal), the AlexNet- CNNs can only accept the inputs in images (2-Dimensional inputs). Therefore, was a need for a data transformation process, from 1-D to 2-D. We applied Continuous Wavelet Transform to convert each ECG signal into scalograms and later were used as the inputs in the proposed model. First, all the selected ECG signals under this study were converted into an image of 227x227. The ECG recordings used in this experiment are the MIT-BIH Normal Sinus Rhythm Database, MIT-BIH Malignant Ventricular Ectopy, and Creighton University Ventricular Tachyarrhythmia database found on the PhysioNet website [27]. From each database mentioned above, we randomly selected 20 ECG signals and then combined them to form a single dataset with a total of 60 ECG records with three different labels as NSR, VF, and VT with equal distribution. To fulfil the requirements of the CNN model, we maximized the size of our database by breaking it into 20 pieces of signal length which gave us 1200 signals in total with 500 samples. The dataset was then separated into two main parts: (i)Training data consists of $75 \%$ of the whole dataset, which carries 1050 signals, and (ii) Validation and Test data was $25 \%$ which is 150 signals. The ratio of the data was equal in all three classes (NSR, VF, and VT).

\subsubsection{Arrhythmia Classification using Convolutional Neural Network}

A pre-trained deep learning neural network called AlexNet was used to detect and distinguish two different arrhythmias (VF and VT) from normal heart rhythms. AlexNet is a Convolutional Neural Network, which has been trained in masses of images to classify other photos into 1000 groups. The AlexNet model has the following features, making it more powerful than other models: The nonlinear activation function, overlapping pooled layers, multiGPU implementation, and local response normalization layer.

In this paper, we did not build a new model from scratch; we referred to the first model of CNN, which was invented in 2012 by Alex Krizhevsky, Ilya Sutskever, and Geoffrey Hinton [28], and applied the transfer learning method. The original model of CNN contains 8 layers, starting with 5 convolutional layers and ending with 3 fully connected layers.

The convolution core sizes in the first layer are $11 \times 11$, followed by $5 \times 5$ in the second layer, and then $3 \times 3$ core sizes. After that, the $1^{\text {st }}, 2^{\text {rd }}$, and $5^{\text {th }}$ convolutional layers are followed by pooled operations with overlapping measures of $3 \times 3$ and steps of $2 \times 2$. In our case, the modification was done only on the last three layers, which are called fully connected layers to suit our goal, while the rest remained unchanged. As shown in Figure 5, the fully connected layer, softmax layer, and classification layer are modified to classify three 
different heartbeats: normal sinus rhythm, ventricular tachycardia, and ventricular fibrillation.

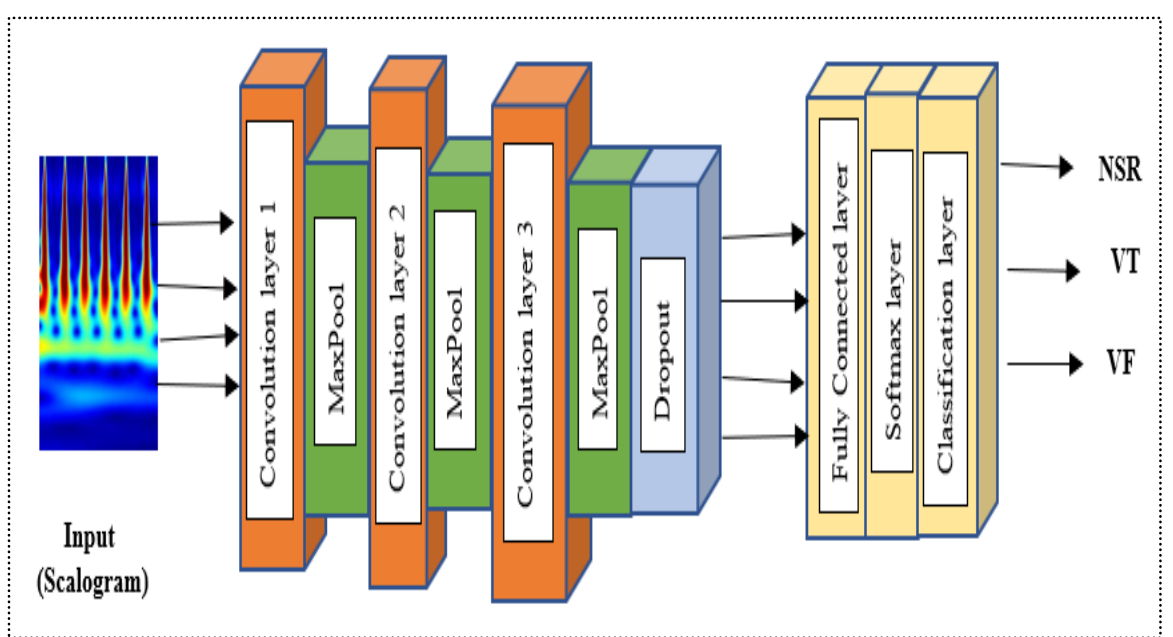

Figure 5. The block diagram of the proposed AlexNet-CNN for arrhythmia classification

\section{EXPERIMENT AND ANALYSIS}

We discovered that all 23 patients who died of sudden cardiac death had aberrant heart rhythms before their deaths; 19 patients had Ventricular fibrillation (VF), 4 patients had Ventricular tachycardia (VT) and none of them had normal heart rhythms (NSR). As a response, we train a pre-trained deep CNN model to detect and distinguish the healthy rhythm (NSR) from the fatal arrhythmias in sudden cardiac death, Ventricular Tachycardia (VT) and Ventricular Fibrillation (VF). Figure 6 depicts some of the patients' ECG signal data when executed on the MATLAB platform.

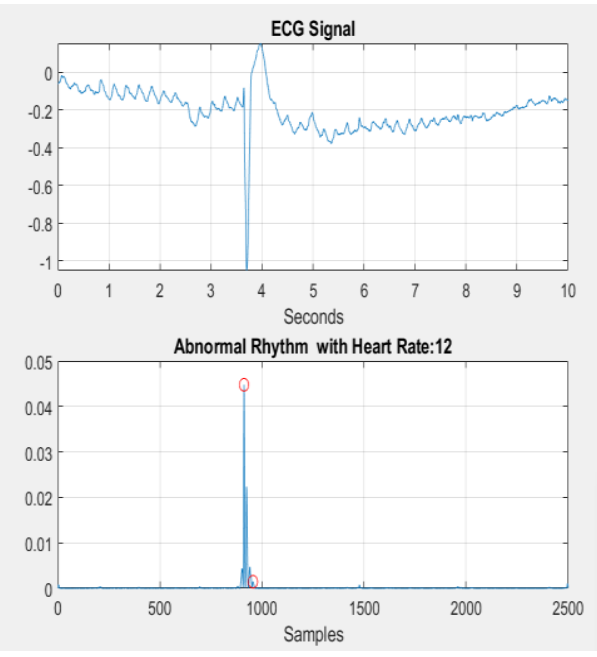

(a)

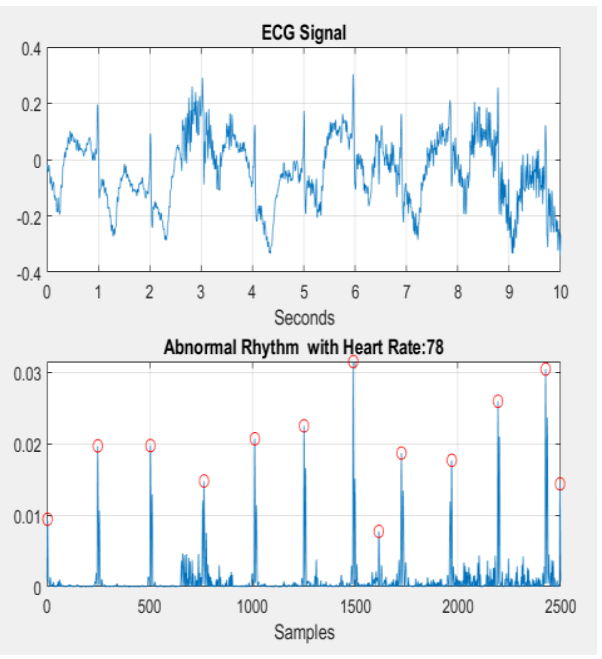

(b)

Figure 6. The experimental results of ECG signals showing the abnormal heart rhythms as executed on MATLAB2018b platform, (a) is record 37m and (b) record 47 from Sudden Cardiac Death Holter database (sddb) database 
The proposed system achieved a profound performance accuracy of 98.7\% and an F-score of 0.9867 when tested in MIT-BIH Normal Sinus Rhythm, MIT-BIH Malignant Ventricular Ectopy, and Creighton University Ventricular Tachyarrhythmia databases. The performance analysis of the proposed model can be evaluated based on the overall accuracy of the system and the true classification verse misclassification per each class, as can be seen in the confusion matrix in figure 7 . Starting with the general performance, our model was correct in classifying the proposed arrhythmias by $98.7 \%$ and was wrong by $1.3 \%$; this means that out of 150 ECG images that were used in testing our model in all three classes (NSR, VT, VF) only 2 images were misclassified. When considering the performance of each class, the results are analyzed as follows; In NSR class, there is no misclassification meaning the system was correct in predicting all of 50 ECG images as NSR, therefore, achieved 100\% accuracy result. In the VF class, out of 50 signals, there is one misclassification entered VT, which gave them $98.0 \%$ accuracy and $2.0 \%$ error. Also, in VT one image was misclassified as VF; therefore, $98.0 \%$ and $2.0 \%$ accuracy and error were achieved, respectively.

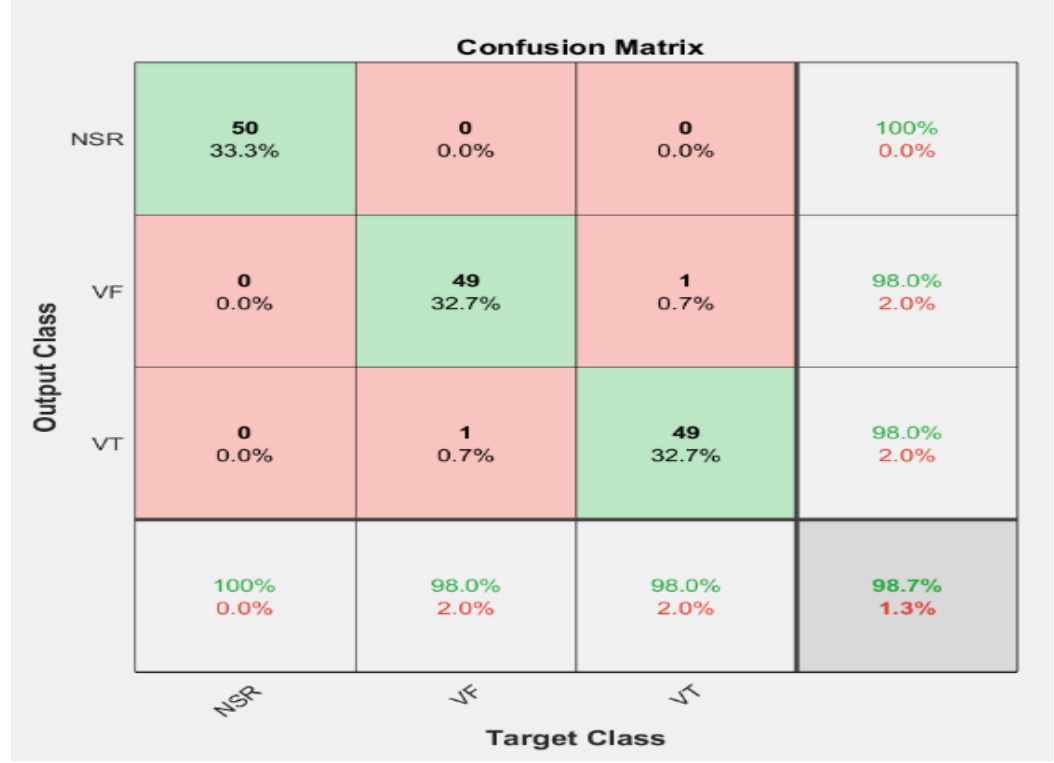

Figure 7. The confusion matrix of the proposed model for arrhythmia classification

In addition, the F-score, also known as the $\mathrm{F}$ measure, was used to test our model's performance as expressed in equation 3 and the value of the Fscore was 0.9867 .

Precision $=\frac{\mathrm{TP}}{\mathrm{TP}+\mathrm{FP}}$
Recall $=\quad \frac{\mathrm{TP}}{\mathrm{TP}+\mathrm{FN}}$


By considering equations 1 and 2 , the equation to calculate the $\mathrm{F}$ measure of our model can be obtained whereby TN is true negatives, TP is true positives, FP is false positives, and FN is false negatives. Thus, the F measure combines recall and precision into a single bar, as indicated in equation 3 .

\section{F-score $=\underline{2 * \text { Precision } * \text { Recall }}$ \\ Precision + recall}

Our system worked well in the NSR class but failed to differentiate two scalograms from the VF and VT classes, according to the findings of the studies. The misclassification resulted from those two images sharing the same characteristics (colour, size, and form) but having distinct classes. Furthermore, figure 8 shows the accuracy and loss graph, which shows that the system remained flat after 7 epochs, indicating that the classification accuracy would not improve even if the number of epochs was increased. As a result, the only way to increase the accuracy of our model is to deal with the misclassification data. In terms of learning patterns, extracting features, and categorizing images, $\mathrm{CNN}$ is outstanding. However, it cannot distinguish data from distinct identities with comparable attributes. As a result, to improve our system's categorization accuracy. First, we suggest looking into the details of each misclassified image from the VF and VT classes and analyzing their similarities and differences. Then, using the misclassification data received, train the classifiers and create new image representations by integrating the classifiers' outputs with CNN to conduct fine-grained image classification. Finally, the classification accuracy will be improved by combining fine-grained image classification approaches with WT-CNN's suggested method. Finegrained classification is nothing but the distinction between difficult-toidentify object classes or the classification of items that are difficult to distinguish due to their similarities [29].

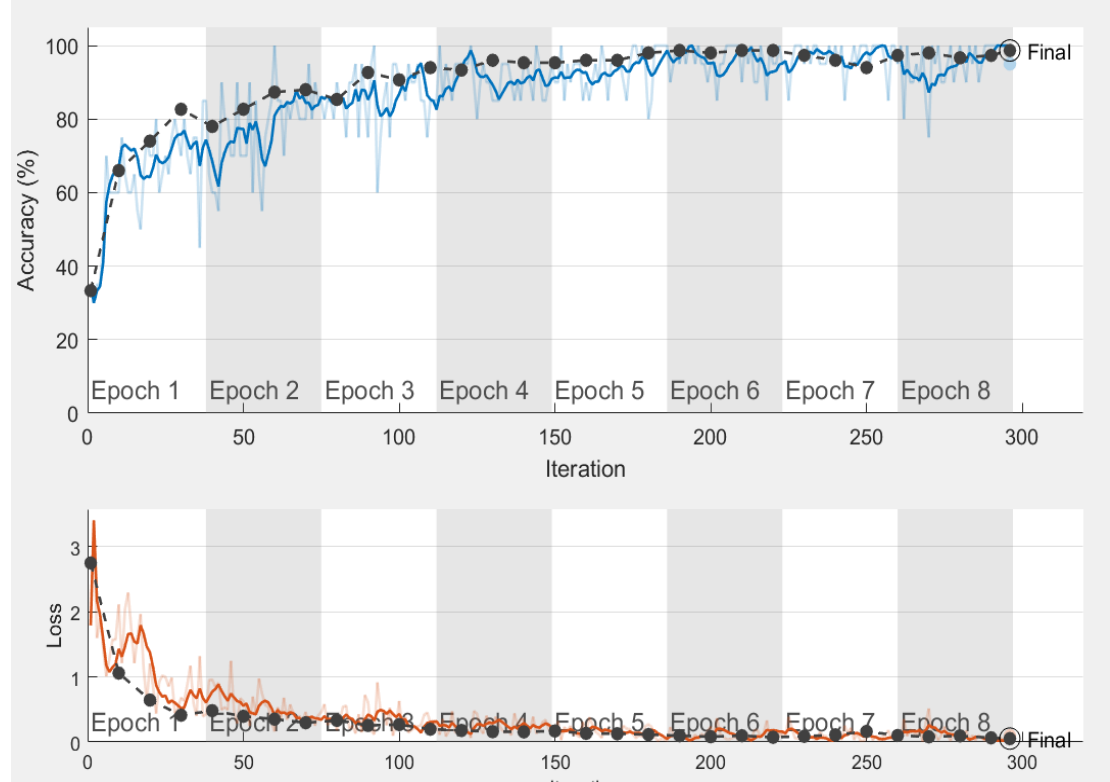

Figure 8. Graph showing the accuracy and loss of the proposed system 


\section{CONCLUSION}

This work investigated 23 ECG records of the patients who died due to sudden cardiac death (SCD) and found that all patients manifested abnormal rhythms or cardiac arrhythmias before their deaths. Then we utilized the Wavelet Transform (WT) and a pre-trained Deep Convolutional Neural Network (CNN) to classify the most lethal arrhythmias in sudden cardiac death, VF, and VT from NSR. WT was used to convert an ECG signal into a scalogram and CNN for features extraction and arrhythmias classification. A well-recognized database: MIT-BIH Normal Sinus Rhythm and MIT-BIH Malignant Ventricular Ectopy were used to examine our model. Even though our model is less costly and noncomplex in implementation achieved profound results: accuracy $=98.67 \%$ and an F-score $=0.9870$. Therefore, the proposed method can be considered in the early detection of arrhythmias for mortality reduction. Since morbidity and mortality of Sudden Cardiac Death increase worldwide very fast. A well-designed disturbance-free cardiac arrhythmia monitoring system for the long term and continuous heart tracking is needed to save lives. So far, a deep learning machine, particularly CNNs, has made an enormous contribution to the investigation of medical data by providing outstanding results in diagnosing and detecting diseases. However, more studies are needed to improve the performance of these algorithms for better treatments.

Patients with a family history of SCD and heart problems are highly recommended for urgent medical attention with continual electrocardiograph screening. And for the whole population, it is advised to avoid poor lifestyle, strenuous exercise, and emotional agitation to help control the problem. The prevention of heart diseases plus early detection of arrhythmias may lead to timely and proper treatment, saving many lives.

\section{REFERENCES}

[1] Kuriachan VP, Sumner GL, Mitchell LB, Sudden Cardiac Death, Curr Probl Cardiol, vol. 40(4), pp. 133-200, April 2015.

[2] Feng X-F, Hai J-J, Ma Y, Wang Z-Q, Tse H-F, Sudden Cardiac Death in Mainland China, Circ Arrhythm Electrophysiol , November 2018

[3] Zhang S, Sudden cardiac death in China: current status and future perspectives, EP Eur, vol. 17(suppl_2), pp. ii14-8, October 2015 .

[4] Zhang J, Zhou X, Xing Q, Li Y, Zhang L, Zhou Q, et al., Sudden cardiac death in the Kazakh and Han peoples of Xinjiang China, Medicine (Baltimore), vol. 18126, pp. 98(50), December 2019.

[5] MA T, A B, W S, G N, A C, KM K, et al., Status of cardiac arrhythmia services in Africa in 2018, a PASCAR Sudden Cardiac Death Task Force report, Cardiovasc J Afr, vol. 29(2), pp. 115-21, 2018.

[6] Chin A, Sudden cardiac death in Africa, Cardiovasc J Afr. , vol. 25(4), pp. 151-2, 2014.

[7] Bonny A, Ngantcha M, Scholtz W, Chin A, Nel G, Anzouan-Kacou J-B, et al., Cardiac Arrhythmias in Africa: Epidemiology, Management 
Challenges, and Perspectives, J Am Coll Cardiol. , vol. 8;73(1), pp. 1009, January 2019.

[8] Sudden Cardiac Death (SCD): Symptoms, Causes , https://my.clevelandclinic.org/health/diseases/17522-sudden-cardiacdeath-sudden-cardiac-arrest

[9] Khan AH, Hussain M, Malik MK, Arrhythmia Classification Techniques Using Deep Neural Network, vol. 20; e9919588, April 2021.

[10] Srinivasan NT, Barts Heart Centre, St Bartholomew's Hospital, London, UK, Schilling RJ, Barts Heart Centre, St Bartholomew's Hospital, London, UK, Sudden Cardiac Death and Arrhythmias,Arrhythmia Electrophysiol Rev, vol. 7(2), pp. 111, 2018.

[11] Assodiky H, Syarif I, Badriyah T, Arrhythmia Classification Using Long Short-Term Memory with Adaptive Learning Rate, Emit Int J Eng Technol, vol. 6(1), pp. 75-91, June 2018.

[12] Moore B, Semsarian C, Chan KH, Sy RW, Sudden Cardiac Death and Ventricular Arrhythmias in Hypertrophic Cardiomyopathy. Heart Lung Circ, vol. 28(1), pp. 146-54, January 2019.

[13] Jazayeri M-A, Emert MP, Sudden Cardiac Death, Med Clin North Am. , vol. 103(5), pp. 913-30, September 2019.

[14] Murugesan B, Ravichandran V, Ram K, S.P. P, Joseph J, Shankaranarayana SM, et al, ECGNet: Deep Network for Arrhythmia Classification, IEEE International Symposium on Medical Measurements and Applications (MeMeA), pp. 1-6, 2018.

[15] Xiong Z, Nash MP, Cheng E, Fedorov VV, Stiles MK, Zhao J, ECG signal classification for the detection of cardiac arrhythmias using a convolutional recurrent neural network, Physiol Meas,vol. 39(9):094006, September 2018.

[16] Wu M, Lu Y, Yang W, Wong SY, A Study on Arrhythmia via ECG Signal Classification Using the Convolutional Neural Network, Front Comput Neurosci, vol. 14, pp. 106, 2021.

[17] Acharya UR, Fujita H, Lih OS, Adam M, Tan JH, Chua CK, Automated detection of coronary artery disease using different durations of ECG segments with convolutional neural network, Knowl-Based Syst, vol. 132, pp. 62-71, 2017 Sep.

[18] Shin H-C, Roth HR, Gao M, Lu L, Xu Z, Nogues I, et al, Deep Convolutional Neural Networks for Computer-Aided Detection: CNN Architectures, Dataset Characteristics and Transfer Learning, IEEE Trans Med Imaging, vol. 35(5), pp. 1285-98, May 2016.

[19] Isin A, Ozdalili S, Cardiac arrhythmia detection using deep learning, Procedia Comput Sci. , vol. 120, pp. 268-75, 2017.

[20] Mashrur FR, Dutta Roy A, Saha DK, Automatic Identification of Arrhythmia from ECG Using AlexNet Convolutional Neural Network, International Conference on Electrical Information and Communication Technology (EICT) Khulna, Bangladesh, IEEE, pp. 1-5 2019. 
[21] Alaskar H, Alzhrani N, Hussain A, Almarshed F, The Implementation of Pretrained AlexNet on PCG Classification, Intelligent Computing Methodologies, Cham: Springer International Publishing, pp. 784-94, 2019.

[22] Ara I, ECG Signal Analysis Using Wavelet Transform, vol. 5(5), pp.7, 2014.

[23] J.Kameenoff, J.Kameenoff, Signal Processing Techniques for Removing Noise from ECG Signals, Biomed Eng Res, vol. 8;1(1), pp. 1, March 2017.

[24] Faust O, Kareem M, Ali A, Ciaccio EJ, Acharya UR, Automated Arrhythmia Detection Based on RR Intervals, Diagnostics, vol. 11(8), pp. 1446, Augast 2021.

[25] Razavi SR, Mohammadi MHD, R-peak Detection in Electrocardiogram Signals Using Continuous Wavelet Transform, vol. 14, 2017.

[26] Velic M, Padavic I, Car S, Computer aided ECG analysis; State of the art and upcoming challenges, Eurocon 2013. Zagreb, Croatia: IEEE, June 2013.

[27] PhysioBank ATM, https://archive.physionet.org/cgi-bin/atm/ATM

[28] Krizhevsky A, Sutskever I, Hinton GE, ImageNet Classification with Deep Convolutional Neural Networks, Advances in Neural Information Processing Systems, Curran Associates, Inc, 2012.

[29] Wang D-H, Zhou W, Li J, Wu Y, Zhu S, Exploring Misclassification Information for Fine-Grained Image Classification, Sensors, vol. 21(12), pp. 4176, January 2021. 